\title{
Platysma Myoides Muscle
}

National Cancer Institute

\section{Source}

National Cancer Institute. Platysma Myoides Muscle. NCI Thesaurus. Code C33329.

A broad, fan-shaped muscle located in the superficial fascia of the neck, extending superiorly from the inferior border of the mandible and inferiorly to the clavicle and the fascia of the anterior shoulder and thorax, that functions to depresses the mandible and wrinkle the skin of neck. 